\title{
Article
}

\section{Tilt Active Vibration Isolation Using Vertical Pendulum and Piezoelectric Transducer with Parallel Controller}

\author{
Lihua $\mathrm{Wu}{ }^{1}$, Yu Huang ${ }^{1,2, *}$ and Dequan $\mathrm{Li}^{1}$ \\ 1 College of Physics and Optoelectronic Engineering, Harbin Engineering University, Harbin 150001, China; \\ wulihua@hrbeu.edu.cn (L.W.); lidequan@ciomp.ac.cn (D.L.) \\ 2 Key Lab of In-fiber Integrated Optics, Ministry Education of China, Harbin Engineering University, \\ Harbin 150001, China \\ * Correspondence: huangyu@hrbeu.edu.cn
}

Citation: Wu, L.; Huang, Y.; Li, D. Tilt Active Vibration Isolation Using Vertical Pendulum and Piezoelectric Transducer with Parallel Controller. Appl. Sci. 2021, 11, 4526. https:// doi.org/10.3390/app11104526

Academic Editor: Giosue Caliano

Received: 2 April 2021

Accepted: 28 April 2021

Published: 15 May 2021

Publisher's Note: MDPI stays neutral with regard to jurisdictional claims in published maps and institutional affiliations.

Copyright: (c) 2021 by the authors. Licensee MDPI, Basel, Switzerland. This article is an open access article distributed under the terms and conditions of the Creative Commons Attribution (CC BY) license (https:// creativecommons.org/licenses/by/ $4.0 /)$.

\begin{abstract}
Tilt vibrations inevitably have negative effects on some precise engineering even after applying horizontal and vertical vibration isolations. It is difficult to adopt a traditional passive vibration isolation (PVI) scheme to realize tilt vibration isolation. In this paper, we present and develop a tilt active vibration isolation (AVI) device using a vertical pendulum (VP) tiltmeter and a piezoelectric transducer (PZT). The potential resolution of the VP is dependent on the mechanical thermal noise in the frequency bandwidth of about 0.0265 nrad, which need not be considered because it is far below the ground tilt of the laboratory. The tilt sensitivity of the device in an open-loop mode, investigated experimentally using a voltage controller, is found to be $(1.63 \pm 0.11) \times 10^{5} \mathrm{~V} / \mathrm{rad}$. To compensate for the hysteresis nonlinearity of the PZT, we experimentally established the multi-loop mathematical model of hysteresis, and designed a parallel controller consisting of both a hysteresis inverse model predictor and a digital proportional-integral-differential (PID) adjuster. Finally, the response of the device working in close-loop mode to the tilt vibration was tested experimentally, and the tilt AVI device showed a good vibration isolation performance, which can remarkably reduce the tilt vibration, for example, from $6.0131 \mu \mathrm{rad}$ to below $0.0103 \mu \mathrm{rad}$.
\end{abstract}

Keywords: tilt active vibration isolation; precise engineering; vertical pendulum; piezoelectric actuator; parallel controller

\section{Introduction}

The vibration caused by a vehicle running, an engine operating, a person walking or an earthquake will have non-negligible and negative effects on precise engineering, such as in measurement experiments and on machining instruments. Generally, in most of the vibration isolation system used in engines, vehicles, etc., the frequency range of an active VIS is from tens to hundreds of $\mathrm{Hz}$ [1]. Various types of microscopes, laser frequency stabilization devices and gravitational wave detection devices usually operate in relatively quiet experimental environments, and these devices will be exciting by low-frequency ground vibrations from a few $\mathrm{Hz}$ to several $\mathrm{Hz}$. Therefore, vibration isolation at these low frequencies is of importance to improve the precision of measurement experiments. The amplitude of ground vibration is very small, generally at the level of $\mu \mathrm{rad}$, so it will not harm people and cannot spread far. The six-degree-of-freedom AVI system in a molecular measuring machine $\left(\mathrm{M}^{3}\right)$ uses five PZTs as the lateral-constraint links in a Mallock suspension system to control the vertical system, which improves the $\mathrm{M}^{3}$ image resolution and measurement speed [2]. A schematic of the lumped model of the atomic force microscope (AFM) system was derived and the vibrational influences of the AFM components were experimentally investigated, and a reconfigured AFM system was constructed and its effects were compared with a conventional system through a series of simulations and experiments [3]. An improved mounting configuration for a passive optical cavity used for laser frequency stabilization was reported, and the associated reduction in the vibration 
sensitivity of the effective cavity length lead to a simple and compact reference cavity system for laser stabilization at the line-width of $1 \mathrm{~Hz}$ [4]. A feed-forward vibration isolation method on a suspended Fabry-Perot interferometer, using Wiener filtering, was reported, with a variant of the least mean square (LMS) adaptive filter algorithm, and the experimental results were compared with theoretical estimations of the attenuation efficiency [5]. To determine the influence of the Raman phase noise, as well as of parasitic vibrations, on the interferometer phase, the sensitivity function of a three-pulse atom interferometer was calculated theoretically and measured experimentally, which determined that the vibration noise would be, by far, the dominant limitation to the sensitivity of the gravimeter [6]. A compact and stable low-frequency AVI system with a commercial voice coil actuator and seismometer was designed and demonstrated to attenuate the vibration noise caused by reflected Ramon beams, and the vertical vibration with a frequency bandwidth $(0.01-10 \mathrm{~Hz})$, measured by the in-loop seismometer, was reduced by up to 500 , as assessed by the PVI platform [7].

For laser interferometric gravitational wave detectors, the vibration isolation of the test mass from ground motions or seismic noise is very significant, especially as regards large, low-frequency motion, due to the resonant modes of some vibration isolators. A laser interferometer combined with a long-period folded pendulum reference was used to measure the $0.3-3 \mathrm{~Hz}$ frequency range horizontal seismic spectrum, and the experiment showed that the horizontal seismic motion of the laboratory was about $10^{-9} \mathrm{~m} / \sqrt{\mathrm{Hz}}$ [8]. To weaken the low-frequency residual tilt vibration, some tilt detectors and servo-loop controllers have been reported and developed. A high-frequency tiltmeter, based on a dumbbell suspended near its center mass, was used to place a preliminary limit on the magnitude of tilts in the laboratory of $1 \mathrm{nrad} / \sqrt{\mathrm{Hz}}$, in the frequency range 3-10 $\mathrm{Hz}$ [9]. The sensitivity obtainable from a kind of optical angular sensor, using the walk-off of a laser between two parallel mirrors, was analyzed theoretically and investigated experimentally [10]. The instrumental noise and transfer function of a double-flexure two-axis tilt sensor with a tilt readout based on an optical walk-off sensor were investigated theoretically and experimentally [11]. The VIGRO GW group used a rotational accelerometer with a sensitivity of $2-3 \mathrm{nrad} / \mathrm{s}^{2} / \sqrt{\mathrm{Hz}}$ for frequencies below $2 \mathrm{~Hz}$ to measure the seismic ground tilt spectrum [12]. Recently, a new rotational vibration isolator with an extremely low resonant frequency of $0.055 \pm 0.002 \mathrm{~Hz}$, consisting of two concentric spheres separated by a layer of water and joined by very soft silicone springs, was reported to reduce rotational vibration noise at all frequencies above its resonance [13].

This paper reports a tilt active vibration isolator using a vertical pendulum (VP) tiltmeter, with a flat elastic spring suspension and PZTs, as a detector of tilt vibration and a servo-controlled actuator, respectively. To extend the dynamic range, the actuator needs to produce a much larger displacement, and therefore the significant dynamic hysteresis caused by the driving process has to be considered carefully. A high-performance piezoelectric servo-controlled actuator is developed through a parallel controller using an inverse-model actuator and a proportional-integral-differential (PID) digital adjuster.

\section{Principle of Tilt Active Vibration Isolator}

The tilt active vibration isolator, in our design, is composed of a tilt-sensitive probe, a capacitive position transducer measuring the tilt motion of the VP, and a piezoelectric servo-controlled actuator driving the VP back to its former central position. The schematic diagram of the tilt active vibration isolator using a VP and PZTs is shown in Figure 1. The VP is a kind of ordinarily structured physical pendulum suspended by a flat elastic spring. The two opposite capacitance plates with a uniform shape are made by plating silver onto the hollow and columned ceramic substrate, and a pair of differential capacitances are formed via the two opposite capacitances, which are separated electrically from both each other and the central pendulum body. The piezoelectric actuator includes two PZTs that are connected via the output of their driving circuit and placed under a screw supporter of the device's base. 


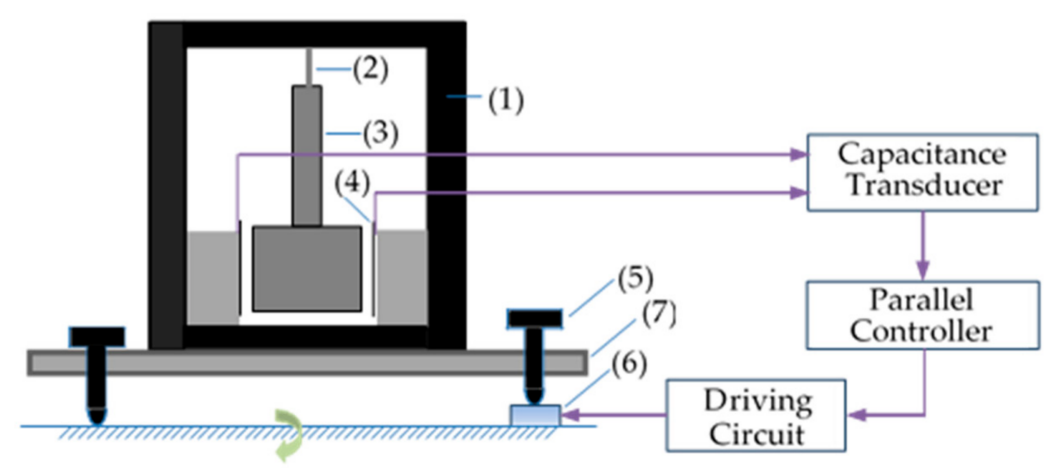

Figure 1. The schematic diagram of the tilt AVI. (1) is the supporting frame, (2) is the flat elastic spring, (3) is the pendulum body, (4) is the capacitance plates, (5) is the adjustable supporting plot, (6) is the piezoelectric actuator, (7) is the plate of the tiltmeter's base.

The VP will depart from its former central position due to tilt vibration acting on the supporting frame. Then, a gap variation between the VP and two capacitances will be produced and measured by a bridge-type capacitance transducer, which is a kind of capacitance sensor, the electric diagram of which is given in Reference 14. To ensure the precise operation of the piezoelectric servo-controlled actuator in a large displacement range, a digital and parallel controller, consisting of a hysteresis inverse model predictor and a PID adjuster, is adopted. This guides the driving circuit to generate the proper voltage to be applied to the two piezoelectric transducers. The digital and parallel controller is implemented by the field programmable gate array (FPGA) in order to easily adjust the controller's parameters. Finally, the VP is driven back to its former central position by the servo-controlled actuator, and the supporting frame also returns to the horizontal state. This means that the supporting frame is immune to the tilt vibration of the ground base. For example, the ground tilting to the right causes the frame to tilt to the right, as shown in Figure 1, and the feedback control circuit drives the PZT to tilt the frame to the left so as to balance the tilt, such that the frame returns to the horizontal state.

\section{Theoretical Analysis of VP}

The structural cross-section of the cylindrical VP is depicted in Figure 2, where point $O$ is the suppression pivot, $l_{1}$ is the effective length of the flat elastic spring, $l_{2}$ and $R_{2}$ are the length and the diameter of the upper solid pole that has a column shape, respectively, and $l_{3}$ and $R_{3}$ are the length and the diameter of the solid cylinder below the pole, respectively.

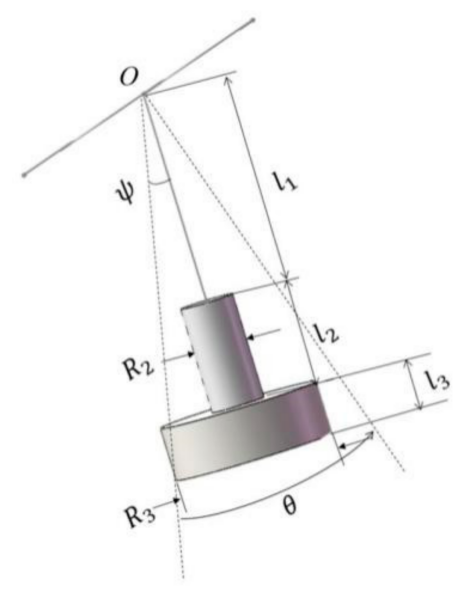

Figure 2. The structural cross-section of the vertical pendulum (VP).

The dynamic equation of the $\mathrm{VP}^{\prime} \mathrm{s}$ response to the tilt vibration $\theta$ is expressed by [14]

$$
I_{O} \ddot{\psi}=-\eta \dot{\psi}-M_{O}+k_{S}(1+j \kappa) \phi
$$


where $I_{O}$, expressed by Equation (2), is the moment of inertia of the VP, $\eta$ is the angular velocity dissipation coefficient caused by the viscous damping resulting from the surrounding air, $\kappa$ refers to the elastic material's inertial friction, $j=\sqrt{-1}$ is an imaginary unit, $\psi$ and $\phi=\theta-\psi$ are the angles at which the vertical pendulum deviates from the vertical section and the frame, respectively, $M_{O}$, expressed by Equation (3), is the gravity torque, and $k_{S}$ is the elastic coefficient of the flat spring, caused by flexural vibration.

$$
\begin{gathered}
I_{O}=\frac{1}{3} m_{2}\left(l_{1}^{2}+3 l_{1} l_{2}+3 l_{2}^{2}\right)+\frac{1}{3} m_{3}\left[l_{3}^{2}+3\left(l_{1}+l_{2}\right) l_{3}+3\left(l_{1}+l_{2}\right)^{2}\right] \\
M_{O}=0.5\left\{m_{2}\left(l_{2}+2 l_{1}\right)+m_{3}\left[l_{3}+2\left(l_{1}+l_{2}\right)\right]\right\} g \psi
\end{gathered}
$$

where $g=9.8 \mathrm{~m} / \mathrm{s}^{2}$ is the gravitational acceleration, and $m_{1}=0.0246 \mathrm{~kg}$ and $m_{2}=0.2953 \mathrm{~kg}$ are, respectively, the masses of the upper and lower solid cylinders of the pendulum's body, $l_{1}=5 \mathrm{~mm}, l_{2}=116 \mathrm{~mm}$ and $l_{3}=40 \mathrm{~mm}$.

Following Equation (1), the function of amplitude transfer, $T_{\phi \theta}(f)$, of the vertical pendulum to ground tilt vibration is expressed by

$$
T_{\phi \theta}(f)=\left|\frac{\phi(f)}{\theta(f)}\right|=\frac{\sqrt{\left(\beta_{0}-\mu^{2}\right)^{2}+\left(\frac{\mu}{Q_{1}}\right)^{2}}}{\sqrt{\left(1-\mu^{2}\right)^{2}+\left(\frac{\mu}{Q_{1}}+\frac{\alpha_{0}}{Q_{2}}\right)^{2}}}
$$

where $\beta_{0}=c /\left(k_{S}+c\right)<1$ is a scale factor representing the mechanical response of the vertical pendulum to low-frequency tilt vibration, $c=0.5\left\{m_{2}\left(l_{2}+2 l_{1}\right)+m_{3}\left[l_{3}+2\left(l_{1}+l_{2}\right)\right]\right\} g$ is a constant with the unit of energy $\alpha_{0}=1-\beta_{0}, Q_{1}=\sqrt{I_{O}\left(c+k_{S}\right)} / \eta$ and $Q_{2}=1 / \kappa$ are, respectively, the quality factor of the mechanical oscillator caused by the viscous damping resulting from the surrounding air and the internal damping within the materials, and $f_{0}=\frac{1}{2 \pi} \sqrt{\left(c+k_{S}\right) / I_{O}}$ is the natural frequency, $\mu=f / f_{0}$. When the vibration frequency satisfies $f \ll f_{0}=\sqrt{\left(c+k_{S}\right) / I_{O}} /(2 \pi), \mu \rightarrow 0$. As $k_{S} \ll c$ and $\alpha_{0} / Q_{2} \ll 1$, in general, for the designed VP, $T_{\phi \theta}(\mu \rightarrow 0) \rightarrow 1$, and this means that the VP undergoes hardly any displacement relative to the vertical direction.

A damped harmonic oscillator undergoes dissipation fluctuation caused by the thermal noise motion of the oscillator at a certain temperature. Thermal noise establishes a significant noise floor within these precise measurements. The mechanical thermal noise of the tilt-sensitive probe is dependent on the loss of the mechanical oscillator, which is due to the viscous damping caused by the surrounding air or the internal damping within the materials. With a vacuum encapsulating the resonating structure, the main limitation is the internal damping within the materials. The thermal noise, based on the fluctuation-dissipation theorem, can be given by [14]

$$
\theta_{t h}(f)=\sqrt{\frac{4 k_{B} T\left(\frac{\mu}{Q_{1}}+\frac{\alpha_{0}}{Q_{2}}\right)}{I_{O}\left(2 \pi f_{0}\right)^{3} \mu\left[\left(\beta_{0}-\mu^{2}\right)^{2}+\left(\frac{\mu}{Q_{1}}\right)^{2}\right]}}
$$

where $T$ is the absolute temperature, and $k_{B}=1.38 \times 10^{-23} \mathrm{~J} / \mathrm{K}$ is the Boltzmann constant.

As the above other parameters are $Q_{1}=8, Q_{2}=12$ and $T=300 \mathrm{~K}$, and the frequency bandwidth is from $0 \mathrm{~Hz}$ to $0.1 \mathrm{~Hz}$, we can calculate the potential resolution of the VP's response to the tilt vibration, as

$$
<\theta_{t h}>=\sqrt{\int_{0}^{0.1} \theta_{t h}^{2}(f) d f}=0.0265 \mathrm{nrad}
$$




\section{Model and Control of PZT}

Actuators, such as the PZT, are widely employed in ultra-precision motion systems because of their distinctive advantages over other driving techniques; for example, their infinite displacement resolution, high stiffness, high actuating force and low heat generation. However, the actuators usually exhibit nonlinear hysteresis behaviors in response to electric fields due to the ferroelectric properties of their materials. Therefore, the voltagedriven strategy based on hysteresis compensation is prevalent in the control of piezoelectric actuators. The improvement of the linearity of the piezoelectric actuator can be realized by implementing model-based compensation strategies.

\subsection{Mathematical Model of Hysteresis}

To improve the driving control accuracy of the piezoelectric actuator with a type of NAC2023 whose resonant frequency is $486 \mathrm{kHz}$, a polynomial model is applied to hysteresis modeling and compensation because of its speed and practicality, and this enables a precise description of the hysteresis phenomenon based on the statistic rule [15,16]. Calibrating the relationship between the displacement and the direct current (DC) driving voltage within the piezoelectric actuator is performed via the optical platform in a stable experimental environment. The driving voltages of the actuator are provided by a piezoelectric power controller, and the displacements are measured by a linear variable differential transformer (LVDT) of the XSI3000 type, whose resolution is about $0.005 \mu \mathrm{m}$. The test and measurement results for hysteresis, which are marked with the symbol "o", and which include rising and descending hysteresis curves denoted by blue and dotted lines, are shown in Figure 3. In Figure 3 , it is obvious that each ascending curve has an inflection point, but the descending curves do not have one. Therefore, we use the third and second polynomials, respectively, to fit the ascending and descending hysteresis curves. For example, the fitting results, denoted by the red and solid lines of the outermost ring, are respectively expressed by

$$
\begin{gathered}
y_{a s 0}=-1.6211 \times 10^{-7} u^{3}+4.7907 \times 10^{-5} u^{2}+0.0122 u-0.0037 \\
y_{d s 0}=-4.1799 \times 10^{-5} u^{2}+0.0213 u+0.1167
\end{gathered}
$$

where $y_{a s 0}$ and $y_{d s 0}$ are the displacement of the actuator in the ascending and descending stages, respectively, and $u$ is the driving voltage of the actuator.

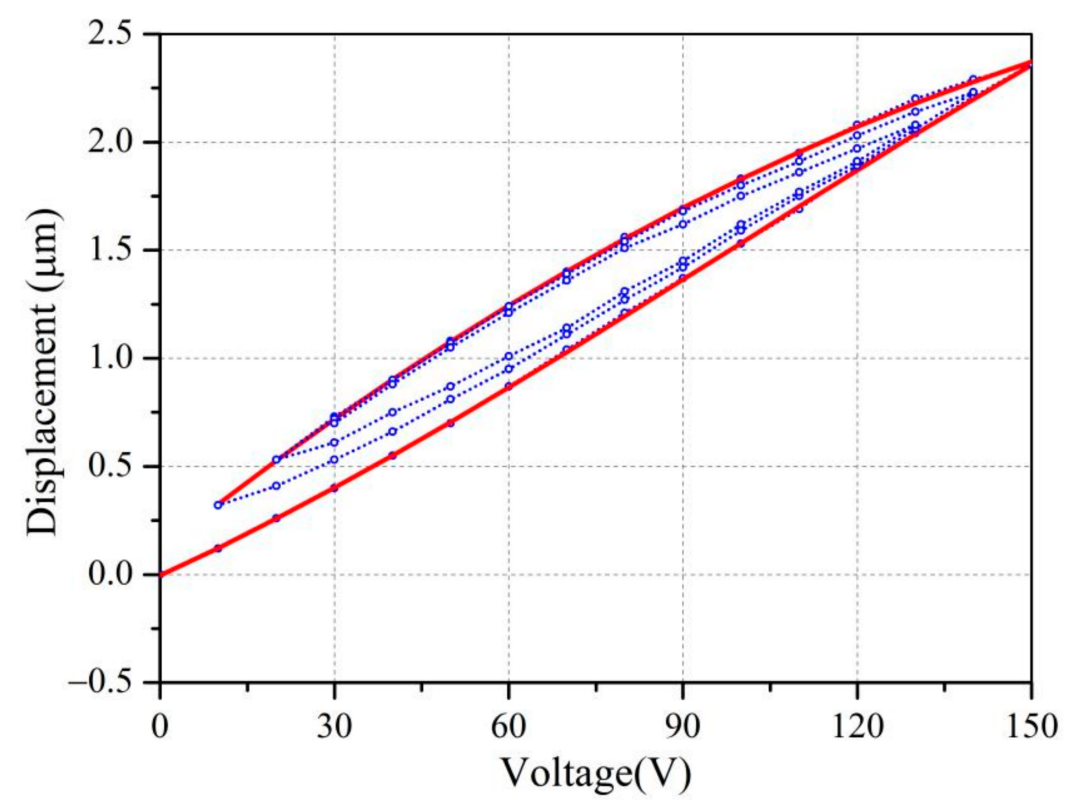

Figure 3. Hysteresis curves of the PZT. 
The results of Figure 3 show that the relationship between the inner ring curves with a smaller range of driving voltages and the outermost ring curve is approximately linear. Therefore, for the descending curves, the relationship of the slope between the inner and outermost rings can be approximately expressed by Equation (9), as follows:

$$
\dot{y}_{d s n}(u)=\dot{y}_{d s 0}\left(u_{s j}+u-u_{0}\right)
$$

where $u_{0}$ is the start voltage of the inner ring's descending curves, $u_{s j}$ is the maximum voltage of the outermost hysteresis, and $y_{d s n}$ is the displacement of the descending curve. The inner descending curve model $y_{d s n}(u)$ and ascending curve model $y_{a s n}(u)$ are established by Equation (9) [15].

The experimental inner loop models are established by the measured ascending and descending displacements, respectively, of the piezoelectric actuator, and these are shown in Figure 4, where the blue lines are the inner loop model curves. The maximum error between the inner loop model and the measured displacement is less than $0.042 \mu \mathrm{m}$.

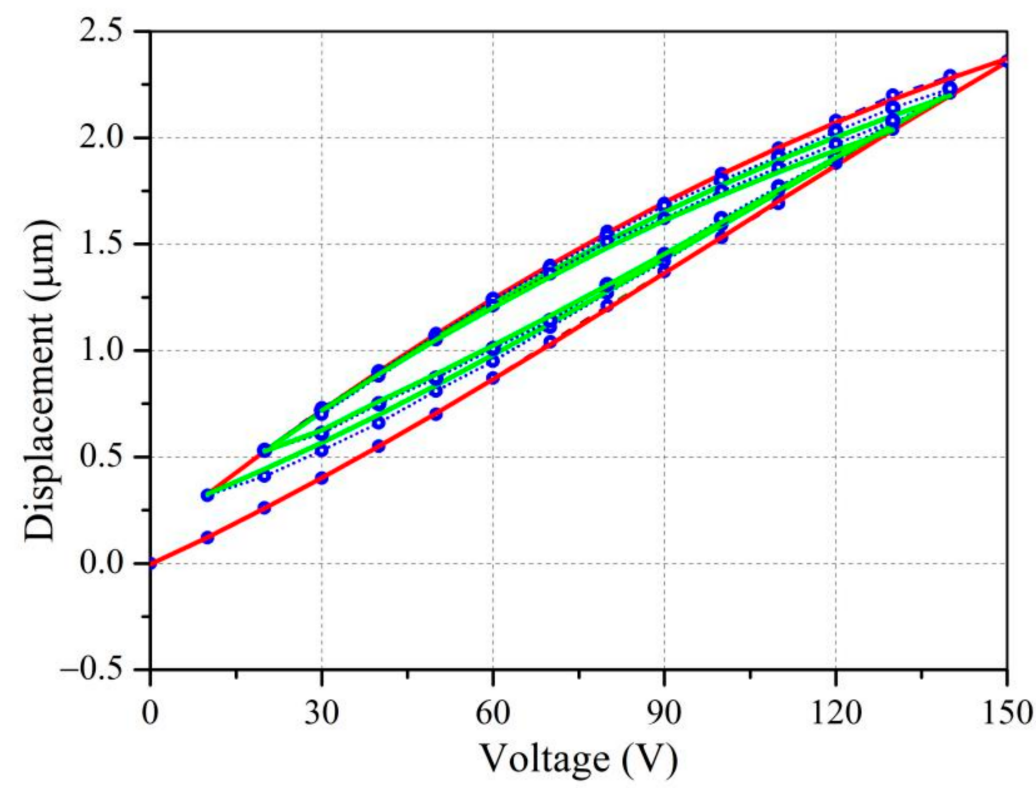

Figure 4. The inner loop model curves of the piezoelectric actuator.

\subsection{Design of Parallel Controller}

The parallel controller for a piezoelectric actuator is based on the prediction of hysteresis nonlinearity using the hysteresis inverse model predictor, and on the digital PID adjuster. The hysteresis inverse model predictor is used to compensate the nonlinearity of the PZT. A block diagram of the tilt vibration isolation control system, composed of a compensation control loop and a PID control loop, is shown in Figure 5. In the close-loop system, an external tilt signal, $\theta$, is compared to the feedback tilt signal, $\theta_{f}$, generated by the piezoelectric actuator, and the VP tiltmeter, including the VP and the capacitance transducer, translates the tilt error, $\Delta \theta$, into the error of displacement, $e_{y}(t)$, which is sampled by the A/D converter. The discrete displacement, $e_{y}(k)$, is simultaneously sent to the digital PID adjuster and the hysteresis inverse model controller, based on the inverse $y_{n}^{-1}$ of the hysteresis model. The digital PID numerical adjuster and the inverse model predictor produce the feedback voltages $\Delta u(k)$ and $u_{c}(k)$, respectively. The A/D converter converts the digital voltage $u(k)$ into the analog voltage $u(t)$, which is sent to the driving circuit of the PZT to produce the aforementioned tilt signal, $\theta_{f}$. 


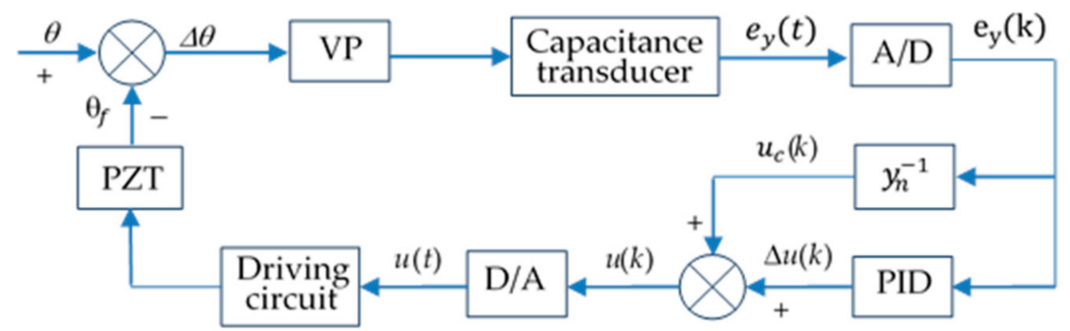

Figure 5. A block diagram of the PID feedback controller with hysteresis model compensation.

The algorithm of the digital PID controller is given by

$$
\Delta u(k)=K_{p}\left\{e_{y}(k)+\frac{T}{T_{i}} \sum_{i=1}^{k} e_{y}(i)+\frac{T_{d}}{T}\left[e_{y}(k)-e_{y}(k-1)\right]\right\}
$$

where $K_{p}$ is the proportional gain, and $T_{i}, T_{d}$ and $T$ are the integral, derivative and sampling time intervals, respectively.

In the loop of the inverse model predictor, it is critical to calculate the compensated voltage, $u_{c}(k)$, according to the error displacement, $e_{y}(k)$. The inverse mathematical models of ascending and descending hysteresis are also solved directly, and the compensated voltage can be given by

$$
u_{c}(k)=\left\{\begin{array}{l}
y_{a s n}^{-1}\left(e_{y}\right), e_{y} \in y_{a s n} \\
y_{d s n}^{-1}\left(e_{y}\right), e_{y} \in y_{d s n}
\end{array}\right.
$$

where $y_{a s n}^{-1}(\cdot)$ and $y_{d s n}^{-1}(\cdot)$ are the inverse functions of $y_{a s n}(\cdot)$ and $y_{d s n}(\cdot)$, respectively.

The additional control voltage, $\Delta u(k)$, is added to the compensated voltage, $u_{c}(k)$, to obtain the total control voltage, $u(k)$, which is used to drive the PZT through the driving circuit.

\section{Experimental Results}

\subsection{Open-Loop Calibration of VP Tiltmeter}

To investigate the response of the VP to tilt, the VP tiltmeter works in an openloop mode without feedback from the piezoelectric actuator. The sensitivity of the VP tiltmeter is calibrated experimentally with a cylindrical piezoelectric transducer of the 20VS12 type, and with a displacement resolution of about $6.3 \times 10^{-4} \mu \mathrm{m}$. The PZT is placed under the base plate, and the screw supporter is adjusted to make the VP close to horizontal. A voltage controller of the XE501D type drives the PZTs to produce three displacement steps of $1 \mu \mathrm{m}$. Figure 6 shows the output curve of the device with a baseline of $165.2 \mathrm{~mm}$ during the calibration, and the parameters $\Delta \mathrm{V}_{1}=(0.97536 \pm 0.00632) \mathrm{V}$, $\Delta \mathrm{V}_{2}=(0.96172 \pm 0.08689) \mathrm{V}$, and $\Delta \mathrm{V}_{3}=(1.02244 \pm 0.09860) \mathrm{V}$ are the three increments of output voltage, respectively. We chose the average of three calibrations as the tilt sensitivity of the VP tiltmeter, so the sensitivity was about $S_{\theta}=(1.63 \pm 0.11) \times 10^{5} \mathrm{~V} / \mathrm{rad}$.

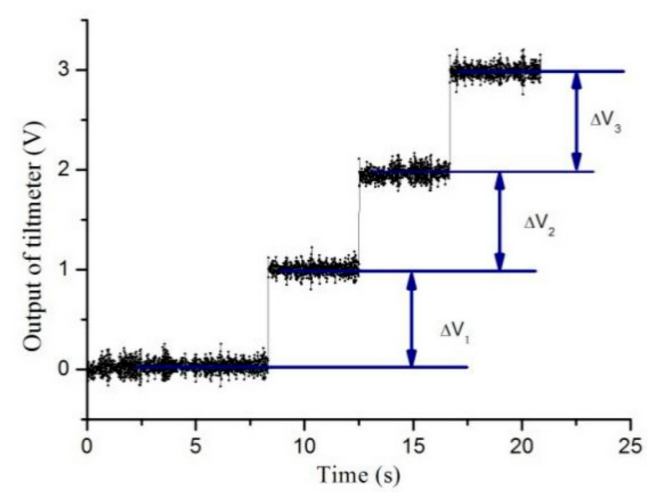

Figure 6. Calibration curve of the device. 


\subsection{Close-Loop Tilt Vibration Isolation}

Firstly, the VP tiltmeter is in the horizontal state, and the cylindrical piezoelectric transducer is extended by lum through the output voltage of the voltage controller to generate a tilt signal to excite the VP tiltmeter, at which point the output data of the VP tiltmeter without feedback are obtained, as is shown in Figure 7. From Figure 7, we can see that the output of the VP tiltmeter is stable at about $1 \mathrm{~V}$.

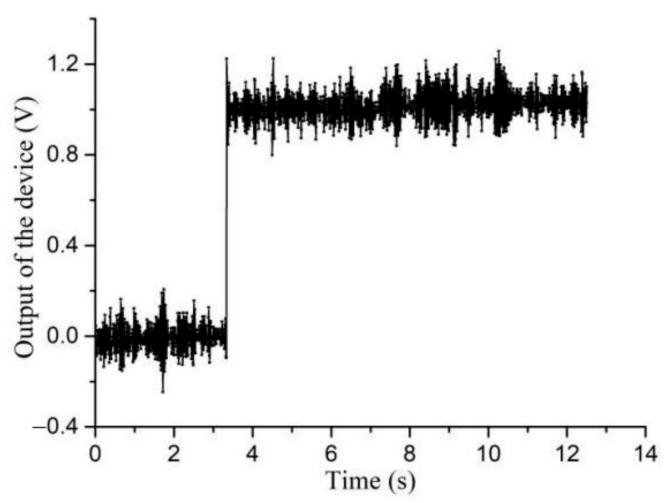

Figure 7. The output of the VP tiltmeter without feedback.

Next, tilt vibration isolation experiments for the device were performed by driving the cylindrical piezoelectric transducer to tilt the supporting base, which is a similar approach to that of the open-loop calibration experiment of the VP tiltmeter. Figure 8 shows the response curve of the device working in close-loop mode to the tilt vibration. To clearly show the tilt vibration isolation procedure, the response from 4.0s to $4.4 \mathrm{~s}$ is shown in the upper right corner of Figure 8. From Figure 8, we can see that the device is firstly horizontal, with an average voltage of $0.02977 \mathrm{~V}$; it then vibrates with the maximum voltage of $0.98013 \mathrm{~V}$, which is consistent with the result of Figure 6, and finally returns to the initial level, with an average voltage of $0.01681 \mathrm{~V}$, in a short time $(\sim 0.067 \mathrm{~s})$. These experimental results demonstrate the tilt vibration isolation of the device under close-loop control, which can reduce the tilt vibration of $\theta_{\text {in }}=6.0131 \mu \mathrm{rad}$ to below $\theta_{\text {out }}=0.0103 \mu \mathrm{rad}$. The damping factor, $\eta_{t d B}$, of the device is $10 \log _{10}\left|\frac{\theta_{\text {out }}}{\theta_{\text {in }}}\right| \approx-27.67 \mathrm{~dB}$.

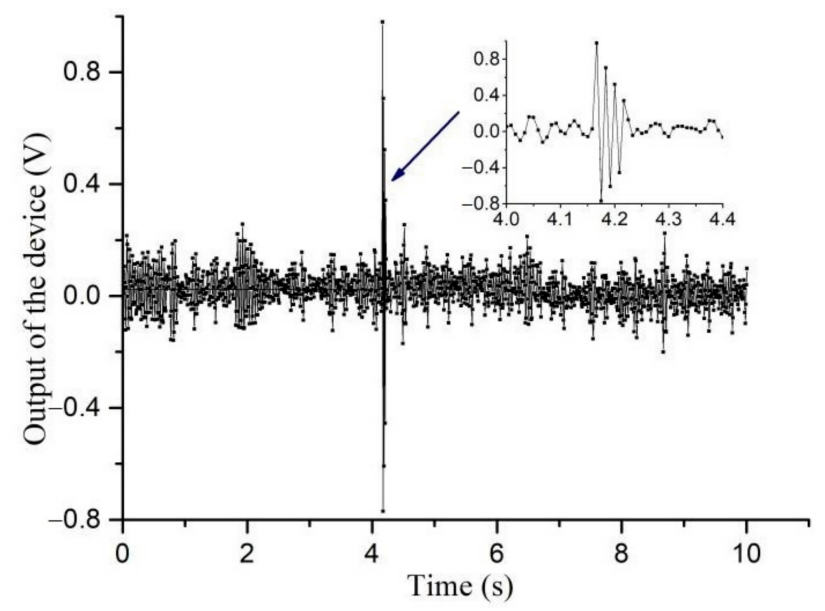

Figure 8. Tilt vibration isolation output of the device under close-loop control.

\section{Conclusions}

The theoretical transfer function of the response to tilt vibration indicates that the VP is sensitive to low-frequency tilt vibrations compared to high-frequency ones. The mechanical thermal noise of the tilt-sensitive VP, due to viscous damping caused by the surrounding air or internal damping within the materials, was evaluated theoretically at 
about $0.0265 \mu \mathrm{rad}$. The sensitivity of the tilt vibro-isolator working in the open-loop mode has been tested experimentally to be about $(1.63 \pm 0.11) \times 10^{5} \mathrm{~V} / \mathrm{rad}$. A kind of parallel controller, consisting of a hysteresis inverse model predictor and a digital PID adjuster, was used to improve the precision of the piezoelectric actuator; the performance of the vibroisolator working in the close-loop mode was investigated, and the experimental results show that the device can reduce the tilt vibration of $6.0131 \mu \mathrm{rad}$ to below $0.0103 \mu \mathrm{rad}$.

Author Contributions: Conceptualization and methodology, L.W. and Y.H.; software, D.L.; validation, Y.H. and D.L.; formal analysis, L.W. and Y.H.; investigation, L.W. and Y.H.; resources, L.W.; data curation, L.W. and Y.H.; writing—original draft preparation, L.W.; writing-review and editing, Y.H.; visualization, D.L.; supervision, Y.H.; project administration, Y.H.; funding acquisition, L.W. All authors have read and agreed to the published version of the manuscript

Funding: This research was funded by [the National Nature Science Foundation of China] grant number [11405035], [the Postdoctoral Science Foundation of China] grant number [2013M530145], [the Postdoctoral Scientific Research Developmental Fund of Heilongjiang Province] grant number [LBH-Q16056].

Institutional Review Board Statement: Not applicable.

Informed Consent Statement: No applicable.

Data Availability Statement: Data sharing not applicable.

Acknowledgments: This work was supported by the National Nature Science Foundation of China under grant numbers 11405035, the Postdoctoral Science Foundation of China under grant numbers 2013M530145, the Postdoctoral Scientific Research Developmental Fund of Heilongjiang Province under grant numbers LBH-Q16056, and the Fundamental Research Fund for the central universities.

Conflicts of Interest: The authors declare no conflict of interest.

\section{References}

1. Yu, Y.; Naganathan, N.G.; Dukkipati, R.V. A literature review of automotive vehicle engine mounting systems. Mech. Mach. Theory 2001, 36, 123-142. [CrossRef]

2. Lan, K.; Yen, J. Sliding mode control for active vibration isolation of a long range scanning tunneling mi-croscope. Rev. Sci. Instrum. 2004, 75, 4367-4373. [CrossRef]

3. Kim, C.; Jung, J.; Youm, W.; Park, K. Design of mechanical components for vibration reduction in an atomic force microscope. Rev. Sci. Instrum. 2011, 82, 035102. [CrossRef] [PubMed]

4. Notcutt, M.; Ma, L.-S.; Ye, J.; Hall, J.L. Simple and compact 1-Hz laser system via an improved mounting configuration of a reference cavity. Opt. Lett. 2005, 30, 1815-1817. [CrossRef] [PubMed]

5. Driggers, J.C.; Evans, M.; Pepper, K.; Adhikari, R. Active noise cancellation in a suspended inter-ferometer. Rev. Sci. Instrum. 2012, 83, 024501. [CrossRef] [PubMed]

6. Cheinet, P.; Canuel, B.; Dos Santos, F.P.; Gauguet, A.; Yver-Leduc, F.; Landragin, A. Measurement of the Sensitivity Function in a Time-Domain Atomic Interferometer. IEEE Trans. Instrum. Meas. 2008, 57, 1141-1148. [CrossRef]

7. Tang, B.; Zhou, L.; Xiong, Z.; Wang, J.; Zhan, M. A programmable broadband low frequency active vibration isolation system for atom interferometry. Rev. Sci. Instrum. 2014, 85, 093109. [CrossRef] [PubMed]

8. Zhou, Z.B.; Yi, Y.Y.; Wu, S.C.; Luo, J. Low-frequency seismic spectrum measured by a laser interferometer combined with a low-frequency folded pendulum. Meas. Sci. Technol. 2003, 15, 165-169. [CrossRef]

9. Speake, C.C.; Newell, D.B. The design and application of a novel high-frequency tiltmeter. Rev. Sci. Instrum. 1990, 61, 1500-1503. [CrossRef]

10. Zhou, Z.B.; Winterflood, J.; Ju, L.; Blair, D.G. Investigation of a laser walk-off angle sensor and its application to tilt measure-ment in gravitational wave detectors. Phys. Lett. 2001, 280, 197-203. [CrossRef]

11. Cheng, Y.; Winterflood, J.; Ju, L.; Blair, D.G. Tilt sensor and servo control system for gravitational wave detection. Class. Quantum Gravity 2002, 19, 1723-1729. [CrossRef]

12. Luiten, A.N.; Beccaria, M.; Bernadini, M.; Braccini, S.; Bradaschia, C.; Cella, G.; Ciampa, A.; Curci, G.; Cuoco, G.E.; D'Ambrosio, E.; et al. Ground tilt seismic spectrum measured with a new high sensitivity rotational accelerometer. Rev. Sci. Instrum. 1997, 68, 1889-1893. [CrossRef]

13. Sunderland, A.; Blair, D.G.; Ju, L.; Golden, H.; Torres, F.; Chen, X.; Lockwood, R.; Wolfgram, P. High performance rotational vibration isolator. Rev. Sci. Instrum. 2013, 84, 105111. [CrossRef] [PubMed]

14. Shu-Chao, W.; Yu, H.; Shu-Hua, F.; Jun, L. Measurement of the Floor Tilt in Experimental Determination of the Gravitational Constant. Chin. Phys. Lett. 2003, 20, 1210-1213. [CrossRef] 
15. Lining, S.; Changhai, R.; Weibin, R.; Liguo, C.; Minxiu, K. Tracking control of piezoelectric actuator based on a new mathematical model. J. Micromech. Microeng. 2004, 14, 1439-1444. [CrossRef]

16. Ru, C.; Sun, L. Improving positioning accuracy of piezoelectric actuators by feed forward hysteresis compensation based on a new mathematical model. Rev. Sci. Instrum. 2005, 76, 095111. [CrossRef] 